\title{
Caractérisation des formations végétales pâturées de la zone guinéenne du Togo : typologie, évaluation de la biomasse, diversité, valeur fourragère et régénération
}

\author{
Kossi Béssan AMEGNAGLO ${ }^{1 *}$, Marra DOURMA ${ }^{1}$, Sêmihinva AKPAVI ${ }^{1}$, \\ Amah AKODEWOU ${ }^{1,2}$, Kpérkouma WALA $^{1}$, Badabaté DIWEDIGA ${ }^{1}$, \\ Wouyo ATAKPAMA ${ }^{1}$, Kodjovi Mawuégnigan Léonard AGBODAN ${ }^{1}$, \\ Komlan BATAWILA ${ }^{1}$ et Koffi AKPAGANA ${ }^{1}$
}

\author{
${ }^{1}$ Laboratoire de Botanique et Ecologie Végétale, Département de Botanique, Faculté des Sciences, Université \\ de Lomé (UL). 01 BP 1515 Lomé 1 Togo. \\ ${ }^{2}$ AgroParisTech (ABIES), CIRAD, Forêts et Sociétés, Montpellier, France. \\ *Auteur correspondant ; E-mail : kossibessan@gmail.com ; Tel :00228 91950333
}

\section{REMERCIEMENTS}

Les auteurs expriment leur gratitude aux Organismes IFS (International Foundation for Sciences) et COMSTECH (Committee on Scientific and Technological Cooperation) qui ont financé ce projet de recherche.

\section{RESUME}

L'élevage contribue à l'économie des populations locales dans les pays en développement, mais reste tributaire de la disponibilité de la végétation naturelle. Le but de cette étude est de caractériser les formations végétales pâturées (FVP) du bassin Mono. Un total de 63 parcelles d'échantillonnage réalisées dans les communautés végétales de la zone ont permis de recenser 311 espèces réparties dans 58 familles et 204 genres. Cinq (05) types de pâturages discriminés sont façonnés par 22 déterminants (topo-édaphiques, anthropogéniques et environnementaux). Le pâturage à Lonchocarpus sericeus et Diheteropogon amplectens $\left(H^{\prime}=5,99\right)$ a l'indice de Shannon le plus élevé tandis que Mitragyna inermis et Cynodon dactylon pâturent l'indice le plus bas $(1,51)$. Les groupes végétaux étudiés sont dominés par les phanérophytes $(43 \%)$ et les thérophytes (34\%). La flore fourragère est dominée par des graminées annuelles (37\%), des graminées pérennes $(23 \%)$ et des graminées vivaces (12\%). Les graminées moyennes et les légumineuses pauvres constituent la majeure partie de la flore fourragère de la zone. La régénération naturelle des ligneux dans les aires de pâture est faible et est due aux impacts des déterminants écologiques et topo-édaphiques. Cette étude servira d'outil d'évaluation et de suivi de la biomasse des FVP dans ces écosystèmes.

(C) 2018 International Formulae Group. All rights reserved.

Mots clés : Biomasse, diversité, paysages pastoraux, caractérisation, régénération, Togo.

\section{Characterization of grazing plant communities in the guinean zone of Togo: typology, biomass assessment, diversity, forage value and regeneration}

\section{ABSTRACT}

Livestock contributes to the economies of local populations in developing countries, but remains dependent on the availability of natural vegetation. The aim of this study was to characterize the grazing plant formations (FVP) of the Mono basin. A total of 63 sampling plot conducted in the plant communities of the 
area identified 311 species in 58 families and 204. Five (05) types of discriminated pastures are shaped by 22 determinants (topo-edaphic, anthropogenous and environmental). Lonchocarpus sericeus and Diheteropogon amplectens grazing land with $\left(\mathrm{H}^{\prime}=5.99\right)$ has the highest Shannon index while Mitragyna inermis and Cynodon dactylon pasture the lowest index (1.51). Studied plant communities were dominated by phanerophytes $(43 \%)$ and therophytes (34\%). The forage flora is dominated by annual grasses (37\%), perennial grasses (23\%) and short perennial grasses (12\%). Middle grasses and poor leguminous make up the bulk of the area-wide forage flora. Natural regeneration of woody in grazing areas is low and is due to the impacts of ecological and topoedaphic determinants. This study will serve as evaluation and monitoring tools for the biomass of the Mono Basin grazing ecosystems.

(C) 2018 International Formulae Group. All rights reserved.

Keyswords: Biomass, diversity, grazing landscapes, characterization, regeneration, characterization, regeneration, Togo.

\section{INTRODUCTION}

Les écosystèmes naturels constituent les principaux pôles pourvoyeurs des biens et services pour l'humanité à travers le monde (MEA, 2005; Brockerhoff et al., 2017; Rolando et al., 2017). Des forêts claires jusqu'aux prairies en passant par les savanes, les formations végétales demeurent des sources diversiformes d'approvisionnement en services écosystémiques pour la survie de l'homme. Ainsi, l'alimentation du cheptel ruminant est l'un des principaux services fournit par la diversité végétale dans les zones à faibles revenus à travers le monde (Sala et al., 2017). Principales sources d'alimentation pour le cheptel ruminant, les formations boisées et herbacées sont assujetties par les systèmes pastoraux pour l'alimentation du cheptel dans les zones tropicales (Correra, 2006; Bechir et al., 2009; Djenontin, 2010).

En Afrique, outre l'agriculture, l'élevage constitue l'un des secteurs dont l'économie reste inféodée aux services des formations végétales. Ce dernier contribue au PIB agricole des pays en développement. Particulièrement dans la zone ouest africaine, l'élevage occupe une part importante dans le développement socio-économique des populations rurales. Environ 150 millions de ménages ruraux vivent au dépens de l'élevage durant toute ou une partie de l'année en Afrique (CEDEAO, 2008; Union africaine, 2010; Krätli et al., 2013a).

Cependant, dans la zone ouest africaine, l'alimentation du cheptel ruminant reste tributaire de la disponibilité spatiotemporelle des formations végétales naturelles. Les cheptels vivent quotidiennement aux dépens des formations végétales naturelles. Ces besoins fourragers ont induit les principales formes de mutations paysagères observables dans les formations végétales pâturées et constituent les signatures de l'impact anthropiques sous toutes ses formes. Cette dynamique a conduit à plusieurs travaux de recherche portant sur les formations végétales de plus en plus en proie à la dégradation (Gregor, 2001). Ces aires de pâture sont de plus en plus confrontées au problème de surpâturage où les populations rurales sont tributaires de l'économie de l'élevage (Fao, 2011). Disponible fourrager pour le cheptel ruminant, ces formations végétales sont de investies au profit de l'élevage locale et par les transhumants transfrontaliers pour leur besoins socioéconomiques (Krätli et al., 2013a) et l'adaptation aux changements climatiques (Kiema et al., 2012; Toutain et al., 2012).

Ces dernières décennies, l'étude des pâturages naturels ont connu une avancée significative en raison leur importance socioéconomique et écologique et écosystémique. Des travaux sur l'écologie, la structure et la dynamique des végétations naturelles pâturées ont été conduits dans la zone ouest africaine (Lejoly and Sinsin, 1991; Gaoue and Sinsin, 2003 ). Aussi, ces ressources fourragères de ces formations ont fait l'objet de recherche en zone soudanienne et sahélienne en l'Afrique (Sinsin, 1993; Djenontin, 2010; Demakou et al., 2011; Demakou et al., 2012; Yameogo et al., 2014). D'autres investigations ont été menées sur ces formations naturelles pour 
évaluer les indicateurs du potentiel fourrager de ces dernières en vue de leur gestion durable (Agonyissa and Sinsin, 1998). Cependant, plusieurs facteurs tels que la composition floristique, la typologie du fourrage, la régénération, etc. restent des indicateurs biophysiques clés nécessitant des investigations supplémentaires en vue de mieux renseigner l'évolution structurelle des formations naturelles pâturées.

$\mathrm{Au}$ Togo, ces formations végétales naturelles sont sujettes aux activités anthropiques surtout d'origines pastorales locales exacerbées par la transhumance transfrontalière (Sokemawu, 2008). Durant toutes les saisons sèches, outre le cheptel local environ 59\% du cheptel bovin ouest africain arrivent au Togo à la recherche de pâturages (Direction des Statistiques Animales du Burkina Faso, 2008). Mais face aux aléas climatiques, la population locale est confrontée aux destructions des récoltes et une pression accrue sur le fourrage ligneux (abattage systématique du fourrage ligneux) au moment où les résidus post récolte n'apportent qu'une faible valeur nutritive aux animaux. Ce déficit du disponible fourrager génère des déséquilibre biologique, socioéconomique, écologique et porte à faux les potentiels écosystémiques et de gestion des écosystèmes pâturés allochtones. Pourtant, les investigations sur les formations végétales pâturées et les ressources fourragères sont obsolètes (Sinsin, 1995). La structure, l'écologie, la typologie, la régénération de ces nouveaux pâturages naturels et les connaissances locales en matière de gestion de ces ressources pastorales et ne sont pas documentées. Ceci nécessite une mise à jour des données sur ces aires de pâture en vue de fournir des indicateurs et pistes de leur gestion durable. C'est dans ce but que cette étude a été entreprise pour une gestion durable des différentes formations végétales pâturées dans la plaine guinéenne du Togo afin d'orienter les décideurs internationaux, nationaux et locaux dans la gestion actuelle de la transhumance et ces problèmes connexes. Les objectifs spécifiques sont (i) étudier la typologie et la diversité floristique de ces formations de la zone ; (ii) analyser les déterminants biophysiques et écologiques directeurs de la diversité floristique des formations végétales pâturées; et (iii) étudier le potentiel de régénération de ces parcours pastoraux.

\section{MATERIEL ET METHODES Zone d'étude}

La présente étude a été conduite dans le sud-est du Togo (Afrique de l'Ouest) (Figure 1). Elle est située dans la partie ouest des régions administrative Maritime et Plateau. Elle est localisée dans le bassin du Mono et couvre une partie des zones humides où l'élevage est de plus en plus développé. La zone d'étude couvre la partie est des zones écologiques III et V (Ern, 1979). C'est une zone localisée dans un ensemble de réseau hydrographique temporaire ou permanent, caractérisée par un important potentiel herbacé fourrager disponible tout au long de l'année et qui devient un nouveau pôle attractif en fourrage pour les éleveurs locaux et les transhumants transfrontaliers. La zone d'étude est limitée au nord par la préfecture de Tchamba, au sud par l'océan atlantique, à l'ouest par la partie ouest des régions Maritime et du Plateau et à l'est par la république du Bénin. Elle est drainée par un système de réseau hydrographique temporaire et permanent (fleuve Mono et affluents Ogou et Anié). La zone abrite également des lacs et un complexe fluvio-lagunaire situé dans sa partie côtière, pôles d'attraction des éleveurs. Elle couvre la partie ouest des zones écologiques III et V (Ern, 1979), et caractérisée par des formations naturelles denses sèches (Adjonou et al., 2010), de forêts communautaires et de mangrove (Afidégnon, 1999) et des reliques forestières (Kokou et Kaballé, 2000). Ces formations naturelles sont réparties dans les aires protégées et dans les zones humides (Adjonou et al., 2010). On y rencontre également une mosaïque de savanes arborées, arbustives, des prairies, des plantations, des parcs, des jachères, etc. L'agriculture et l'élevage restent les principales activités génératrices de revenus des populations locales. La zone accueille environ 1033664 têtes de bovins et 3 
millions de petits ruminants durant ces 10 dernières années (Ministère de l'Agriculture et de l'Elevage, 2011). Les pratiques agropastorale et pastorale, et les feux anthropiques demeurent les principales formes de pressions sur les ressources paysagères et les formations végétales pâturées.

\section{Collecte de données}

Dans le but d'analyser la diversité floristique et la typologie des formations végétales qui servent de source d'alimentation aux cheptels ruminants, des inventaires phytosociologiques y ont été réalisés suivant la méthode de (Braun-Blanquet, 1932). Ces inventaires ont été orientés par les indices de pâturage dans les formations végétales. Les points d'échantillonnage sont définis après une phase exploratoire de visite de terrain couplée à des entretiens avec les populations allochtones dans les zones d'accueil et de transit du cheptel ruminant. Une carte thématique de transit et des itinéraires de transhumance établie par le Ministère de l'agriculture a été aussi utilisée dans le choix des sites d'échantillonnage. La nomenclature des formations végétales de la zone est basée sur les formes biologiques de Raunkiaer (Raunkiaer, 1934; Jürgens, 1990), la hauteur des espèces et le recouvrement de la strate herbacée défini par la Conférence de Yangambi (Aubreville, 1957). Au total, 63 relevés floristique, écologique et forestier ont été effectués à l'échelle de la zone d'étude. Ces relevés ont été conduits en juillet, période de maturité des espèces herbacées. Les relevés de végétation ont été conduits à l'intérieur des placettes harmonisées de $30 \mathrm{~m}$ x $30 \mathrm{~m}$ (Shmida, 1984; Guelly et al., 1997; Peet. et al., 1998; Stohlgren, 2007; Folega et al., 2010a; Wala et al., 2012; Diwediga et al., 2015) pour la strate ligneuse et $10 \mathrm{~m}$ x $10 \mathrm{~m}$ pour la strate herbacée (Lesse et al., 2016). Chaque relevé de végétation est focalisé sur l'inventaire des espèces des strates herbacées et ligneuses suivi du recensement des mesures dendrométriques (hauteur, le diamètre à hauteur de poitrine) (Dimobe et al., 2014).

Les perturbations anthropiques (passage du feu, impact du pâturage, coupe de bois, foyer fabrication $\mathrm{du}$ charbon) et écologiques (type de végétation, degré de submersion, type de sol) ont été relevés en présence / absence (Diwediga et al., 2015). Le potentiel de régénération de l'aire de pâture a été étudié à l'intérieur de sous-placettes de 5 $\mathrm{m} \times 5 \mathrm{~m}$ pour chaque placette d'inventaire (Dourma, 2008 ). Seules les espèces ligneuses dont le diamètre est inferieur $5 \mathrm{~cm}$ ont été prises en compte (Sudhakar Reddy et al., 2011). Le nombre et le type de régénération ont été enregistrés lors de ces relevés de végétation. En plus, d'autres paramètres (le $\mathrm{pH}$ et le taux humidité) de chaque site d'inventaire ont été aussi collectés à l'aide d'un testeur de sol. Pour chaque placette d'inventaire, des coordonnées géographiques ont été aussi enregistrées à l'aide d'un capteur GPS. Le type d'écosystème (zone humide ou non) a été noté. Le tassement du sol a été mesuré (présence/absence) pour analyser l'impact du passage du cheptel sur la structure $\mathrm{du}$ sol. Les indices de prélèvement de biomasse à travers la coupe de bois a été enregistré en terme de présence/absence. Afin d'analyser l'impact des variables topoédaphiques sur la distribution des espèces dans les types de végétation, d'autres variables biophysiques telles que l'indice d'aridité (AI), indice d'humidité ou wetness index (WI), altitude par rapport au thalweg (Altacl), indice d'influence hydrographique ou stream power index (SPI) ont été utilisées pour dans les analyses écologiques. Ces paramètres sont dérivés des données d'élévation du terrain (DEM) disponibles sur le site https://earthexplorer.usgs.gov. Grâce au logiciel ArcGis 10.2. 2, ces paramètres ont été extraits aux coordonnées GPS des placettes d'échantillonnage. AI est un paramètre qui indique l'état d'assèchement d'une zone. WI est une variable topographique qui est utilisée pour quantifier l'influence de la topographie sur le système hydrographique et varie suivant la pente. Altacl est un paramètre qui renseigne sur l'altitude d'un point par rapport au niveau le plus bas du relief. SPI est un indice qui indique l'influence du réseau hydrographique sur l'érosion du sol. 


\section{Analyse des données}

Les données floristiques et écologiques ont été saisies et codifiées dans un tableur Microsoft Excel. La méthode d'ordination a été utilisée pour discriminer les principaux gradients écologiques et les descripteurs environnementaux qui définissent la distribution des espèces et des types de formations végétales dans les paysages pastoraux de la zone. Parmi les méthodes d'analyse multivariées existantes, l'ordination par positionnement multidimensionnel non métrique (NMDS) a été adoptée à l'aide du logiciel $\mathrm{R}$ pour analyser la similarité entre les différentes espèces et leur distribution dans les types de végétations. Les groupements végétaux inventoriés ont été discriminées grâce à une analyse en composante principale (ACP) avec lien complet par le biais du package vegan du logiciel R. Les données de régénération ont été codifiées suivant la moyenne du nombre de pied rejet par relevé et par groupements végétaux et soumis à une analyse des variances à un facteur pour vérifier la significativité du taux de régénération entre les groupements discriminés. La discrimination et l'interprétation environnementale des variables biophysique, écologiques et anthropiques ont été performée grâce à une analyse multidimensionnelle non métrique (NMDS). Les indices d'appétence (IS) utilisés dans le cadre de cette étude sont ceux définis pour la flore des régions tropicales africaines (Daget et Poissonet, 2010). Les espèces dont les (IS) ne figurent pas dans cet ouvrage, ont été définis par appréciation locales des éleveurs sur la base de leur préférence pour le cheptel en comparaison avec les espèces aux IS connus.

La richesse spécifique (S) et les indices de diversité de Shannon (H') et d'équitabilité de Pielou (E) des différents groupements végétaux ont été calculés grâce au package « Biodiversity » du projet $\mathrm{R}$.

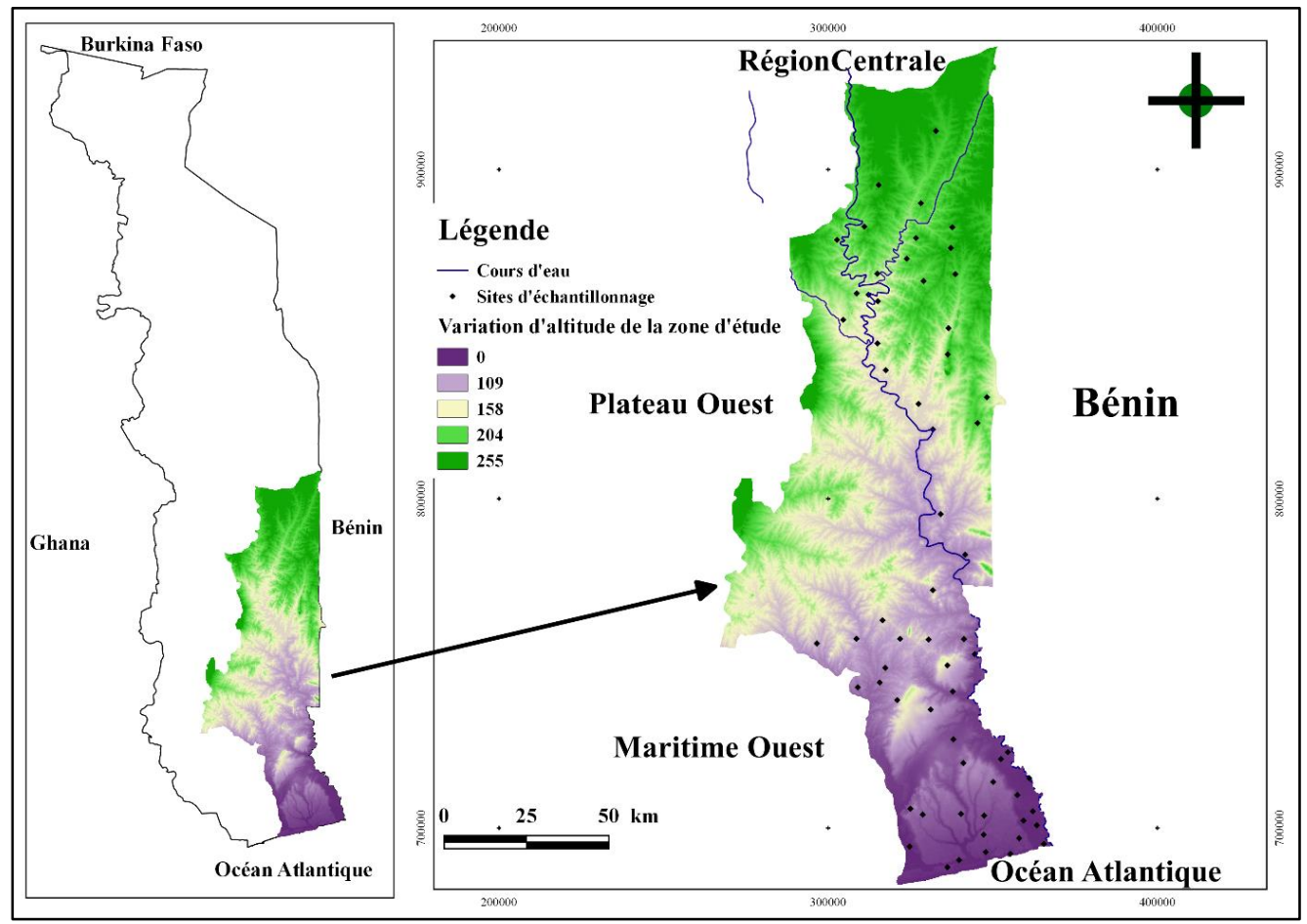

Figure 1 : Zone d'étude et sites d'échantillonnage. 
RESULTATS

\section{Bilan floristique et écologique de la zone Richesse spécifique et spectres des familles et des types biologiques}

Les formations végétales constituent la principale source d'alimentation du cheptel ruminant à travers la zone. La diversité floristique de ces formations reste le principal facteur directeur des mouvements pastoraux. A l'échelle de la zone, les investigations floristiques conduites à travers les pâturages naturels ont permis de recenser 311 espèces. Les familles les plus rencontrées sont les Fabaceae, les Poaceae, les Cyperaceae et les Asteraceae avec respectivement 62, 55, 23 et 15 espèces chacune (Figure 2). L'indice de Shannon est de $H^{\prime}=3,5$ avec un indice d'équitabilité de Pielou est de $E=0,84$.

La biomasse des pâturages constitue la principale source d'alimentation pour le cheptel de la zone. En effet, la flore des aires de pâture est composée de $76 \%$ d'herbacée et $24 \%$ de ligneux. La biomasse appétée des FVP est constituée essentiellement des herbacées annuelles (37\%), des herbacées pérenne $(23 \%)$ et des herbacées vivaces. Les ligneux sont les moins représentés de la flore avec une fréquence de 24\% (Figure 3).

Le spectre des formes biologiques montre que la florule des pâturages composée de Phanérophytes (43\%), hôtes des Epiphytes Loranthacée recensées; les Thérophytes (Th) $(34 \%)$; des Chameophytes (Ch) et les Hémicriptophytes $(\mathrm{He})$ sont les moins représentés (Figure4).

\section{Bilan fourrager}

$\mathrm{Au}$ total, 131 espèces fourragères réparties en 20 familles et 76 genres ont été relevées lors des investigations de relevé de végétation dans les FVP. Les familles les plus représentées sont les Poaceae, les Fabaceae et les Cyperaceae comportant respectivement 48, 31 et 23 espèces. Contrairement aux types biologiques pour l'ensemble de la flore recensée dominée par les Phanérophytes $(\mathrm{Ph})$ suivi des Thérophytes (Th), la tendance est inversée pour les espèces fourragères qui sont dominées par les Thérophytes (Th) (Figure. 4).

\section{Typologie des pâturages}

Sur la base d'une matrice de 311 lignes et 63 colonnes et de l'abondance des espèces dominantes, cinq (5) types de pâturages ont été discriminés à l'échelle de la zone (Figure 5).

Quatre groupes de relevés G1, G2, G3, G4, G5 ont été discriminés à partir de la DCA qui se présente comme suit :

$>$ Le groupe G1, de 14 relevés caractéristiques des savanes arbustives et jachères des zones marécageuses ;

$>$ Le groupe $\mathrm{G} 2$ composé de 10 relevés effectués dans les formations boisées des plaines sur sol noir argileux ;

$>$ Le groupe G3 contenant 20 relevés, ont été investigués dans les savanes arbustives des plaines humides avec des espèces envahissantes sur sol rouge argileux. ;

$>$ Le groupe G4, de 11 relevés est caractérisé par des jachères des plaines submersibles sur vertisols avec un début d'embroussaillement dû à Chromolaena odorata. ;

$>$ Le groupe G5 comportant 8 relevés caractéristiques des jachères de plaines humides partiellement submersibles en saison sèche dominées par une biomasse herbacée pérenne et vivace.

Les groupes G1, G2, G3 caractérisent des relevés effectués dans les zones humides (inondés) et non humides. Ces derniers sont discriminés suivant un gradient d'humidité du sol. Les groupes G4 et G5 regroupent des relevés réalisés dans des jachères humides constamment brûlées. Ce regroupement est influencé par un gradient d'humidité du sol et d'anthropisation

En somme, cinq (05) types de formations végétales pâturées (FVP) ont été identifiés durant les relevés de végétation: 
- $\quad$ Le pâturage à Mitragyna inermis et

Cynodon dactylon (G1) ;

- $\quad$ Le pâturage à Anogeissus leiocarpa et à Panicum maximum (G2) ;

- $\quad$ Le pâturage à Mitragyna inermis et Paspalum orbiculare (G3) ;

- $\quad$ Le pâturage à Lonchocarpus sericeus et Diheteropogon amplectens (G4) ;

- $\quad$ Le pâturage à Machaerium lunatum et Leersia hexandra (G5).

Caractérisation des différents types de FVP

\section{Pâturage à Mitragyna inermis et Cynodon dactylon}

Ce sont des formations arbustives inféodées aux zones humides sur sol noir sablo argileux à hydromorphie permanente. Elles sont constamment soumises aux impacts des feux d'origine anthropiques. Ces formations regroupent 61 espèces réparties en 23 familles 55 genres. Les familles les plus représentées sont les Poaceae, les Fabaceae et les Cyperaceae. Deux strates ont été identifiées sur ces aires de ces aires de pâtures notamment la strate arbustive dominée par Mitragyna inermis, Acacia auriculiformis, Drepanocarpus lunatus, Phoenix reclinata et une strate herbacée caractérisée par Cynodon dactylon; Paspalum orbiculare; Cyperus articulatus. L'indice de diversité de Shannon de ce groupe est 1,51 et celui d'équitabilité de Pielou est de 0,37.

\section{Pâturage à Anogeissus leiocarpa et à Panicum maximum}

Il comprend 10 relevés réalisés dans les formations végétales boisées (forêt claire et savane boisée) des plaines sur sol noir argileux avec une strate herbacée dominée par les espèces pérennes. On y observe des perturbations anthropiques liées au passage fréquent des feux de végétation. Les relevés de végétations de ce pâturage révèlent une florule de 69 espèces répartie en 22 familles et 59 genres. Les Fabaceae (30 espèces); les Poaceae (20 espèces) et les Combretaceae (19 espèces) sont les familles les plus représentées. Deux principales strates se distinguent dans ces formations. Une strate arborée dominée par des essences comme: Anogeissus leiocarpa; Combretum micrantum et Spondias mombin et strate herbacée est dominée par Panicum maximum, Diheteropogon amplectens; Brachiaria mutica et Aeschynomene uniflora. L'indice de Shannon (H') de ce pâturage est de 2,83 et celui d'équitabilité de Pielou 0,36.

\section{Pâturage à Mitragyna inermis et Paspalum orbiculare}

C'est un pâturage constitué de 20 relevés caractéristiques des formations arbustives dans les plaines humides fréquemment soumis aux actions des feux d'origine anthropiques. Il est composé d'une diversité floristique de 169 espèces regroupé en 40 familles et 133 genres. Les Poaceae, les Fabaceae et les Rubiaceae sont les familles les plus représentative et regroupent respectivement $68 ; 61$ et 22 espèces en leur sein. On y distingue une strate arbustive caractérisé par des espèces comme Antidesma venosum, Cassia sieberiana; Elaeis guineensis et une strate herbacée dominée par Paspalum orbiculare; Cynodon dactylon; Sporobolus pyramidalis. L'indice de diversité de Shannon de ce groupement à Mitragyna inermis et Paspalum orbiculare est de 2,97 avec un indice d'équitabilité de Pielou de 0,29 .

\section{Pâturage à Lonchocarpus sericeus et Diheteropogon amplectens}

Il regroupe 11 relevés réalisés dans des formations végétales anthropisées (jachères) souvent brûlées des plaines en phase d'envahissement par Chromolaena odorata et présentant un faible degré de submersion. La flore recensée présente une diversité de 128 espèces regroupées 34 familles et genres. Les Fabaceae sont les familles les plus représentées avec 63 espèces suivies des 
Poaceae 32 espèces et des Combretaceae qui comptent 24 espèces. Les relevés de végétation révèlent une de Shannon de 5,99 et celui d'équitabilité de Pielou est de 0,31. La strate arbustive investiguée est dominée par Annona senegalensis; Combretum collinum; Crossopteryx febrifuga; Daniellia oliveri. Quant à la strate herbacée, elle est caractérisée par une flore herbacée pérenne essentiellement composée de Diheteropogon amplectens ; Digitaria leptorhachis ; Sporobolus pyramidalis et Andropogon gayanus.

\section{Le pâturage à Machaerium lunatum et Leersia hexandra}

Il est constitué de 8 relevés caractéristiques des jachères et savanes arbustives inféodées aux plaines humides partiellement submersibles en saison sèche et constamment brûlées. Les inventaires floristiques révèlent une florule de 143 espèces réparties en 35 familles et 111 genres. Les familles les plus représentées sont les Fabaceae; les Poaceae et les Cyperaceae qui regroupent respectivement $45 ; 30 ; 19$ espèces chacune. La strate herbacée de ce pâturage est dominée par Leersia hexandra ; Acroceras zizanioides; Pennisetum polystachion; Kyllinga bubosa et la strate ligneuse est caractérisée par Machaerium lunatum; Combretum collinum; Pseudocedrela kotschyi; Zanthoxylum zanthoxyloides

\section{Spectre des types fourragers des groupements discriminés}

La typologie des groupements végétaux discriminés montre une diversité de types floristiques inventoriés à travers la zone (Figure. 6). Chaque rectangle représente un type floristique suivant les types de pâturage ou groupe. Sa largeur correspond à son pourcentage selon le pâturage et la couleur de la case correspond aux résidus du test de Chi 2 $\left(\chi^{2}\right)$ correspondant : les cases en rouge sont sous-représentées, les cases en bleu surreprésentées, tandis que les cases blanches sont statistiquement proches de l'hypothèse d'indépendance. Le spectre des pâturages à Mitragyna inermis Cynodon dactylon (G1); à Anogeissus leiocarpa, Panicum maximum (G2) et à Lonchocarpus sericeus Diheteropogon amplectens (G4) et présentent une forte diversité respectivement en herbacées vivaces, en ligneux hauts et en ligneux bas. Ces trois pâturages présentent dans cet ordre une diversité floristique voisine en herbacées pérennes invasives et lianescentes. Le pâturage à Machaerium lunatum et Leersia hexandra (G5) montre, quant à lui, une diversité floristique moyenne avec un tapis graminéen dominé par des herbacées annuelles.

\section{Spectre des types fourragers des groupements discriminés}

Les principaux groupements végétaux discriminés présentent une diversité floristique des types fourragers (Figure 7). Chaque rectangle représente la diversité du type fourrager suivant les différents types de pâturage discriminés. Sa largeur correspond à son pourcentage selon le pâturage et la couleur de la case correspond aux résidus du test de Chi $2\left(\chi^{2}\right)$ correspondant : les cases en rouge sont sous-représentées, les cases en bleu sur-représentées, tandis que les cases blanches sont statistiquement proches de l'hypothèse d'indépendance. Le spectre des types fourragers montre une strate herbacée caractérisée par une forte diversité de graminées moyennes observables dans le pâturage à Mitragyna inermis et Cynodon dactylon (G1). Le tapis graminéen de ce groupement est dominé par une cohorte d'herbacées annuelle, pérenne et vivace. Le pâturage à Anogeissus leiocarpa, Panicum maximum $(\mathrm{G} 2)$ présente une florule fourragère dominée par une diversité fourragère moyenne composée de légumineuses médiocres. Les groupements G1 et G4 enregistre une florule 
très moins diversifiée en graminées moyennes de même que les diverses fourragères dans le groupement à Mitragyna inermis et Cynodon dactylon (G1). Les autres types fourragers présentent une diversité similaire à travers les types de pâturages discriminés.

\section{Ordination partiel des relevés et des espèces}

Variables environnementales directrices de la répartition des relevés et espèces dans les FVP

L'ordination de la double projection (ACP) des déterminants biophysiques et anthropiques enregistrés montre la partition des facteurs directeurs de la distribution des espèces recensées et des relevés à l'échelle de la zone (Figure 8). L'analyse combinée de la PCA présente la coexistence des déterminants biophysiques et les perturbations anthropiques qui partitionnent les différentes composantes environnementales à l'échelle du paysage. La longueur des flèches traduit l'ampleur de l'impact de la variable. Plus la longueur de la flèche est élevée, plus son impact dans la distribution des espèces est grand. Les déterminants en couleur noire influence très significativement la partition des espèces et relevés alors que ceux en bleu n'y ont pas d'influence significative., En effet, suivant l'axe NMDS1, certaines espèces sont inféodées aux relevés où le pâturage, la topographie et l'altitude au niveau des talwegs (altacl) coexistent tandis que d'autres sont tributaires des conditions où l'environnement est soumis au 06 autres déterminants (l'anthropisation, l'infiltration, le degré de submersion, la pédologie, l'occupation du sol et le feu). D'autres espèces préfèrent les conditions caractéristiques d'un gradient d'humidité croissante matérialisées dans le plan factoriel NMDS2.

Evaluation de la régénération dans les différents pâturages

La Figure 9 montre la variation du taux de régénération dans les différents pâturages discriminés à travers la zone. Le taux de régénération dans le pâturage à Anogeissus leiocarpa et Panicum maximum reste le plus important suivi par celui du pâturage à Mitragyna inermis Cynodon dactylon et à Mitragyna inermis Paspalum orbiculare. La régénération dans le pâturage à Machaerium lunatum Leersia hexandra reste faible et celle dans le pâturage à Lonchocarpus sericeus et Diheteropogon amplectens encore plus faible. Le taux de régénération dans chaque type de pâturage a varié aussi sensiblement.

Dans $25 \%$ des cas les ligneux du pâturage à Mitragyna inermis et Cynodon dactylon ont un taux de régénération de 4 ; $50 \%$ avoisine $6 ; 75 \%$ ont un taux de 8 et moins de $100 \%$ ont un taux de 14 . Par contre, $50 \%$ du taux de régénération dans le pâturage à Anogeissus leiocarpa Panicum maximum avoisine 8 et $75 \%$ tend vers 10 . Quant au pâturage à Mitragyna inermis Paspalum orbiculare, $25 \%$ du taux est en bas de $4 ; 50 \%$ en bas de $6 ; 75 \%$ est autour de 8 et plus de $75 \%$ au tour de 10. Dans le pâturage à Lonchocarpus sericeus Diheteropogon amplectens, 50\% de ce taux avoisine 3 alors que plus de $50 \%$ est entre 4 et 7 . Contrairement aux autres pâturages, le taux de régénération varie très peu dans le pâturage à Machaerium lunatum et Leersia hexandra. La variation de ce taux dans $25 \%$ des cas est très négligeable. Mais par contre, $50 \%$ de ce taux dépasse 4 et plus de $50 \%$ avoisine 7 . Les espèces à fort potentiel de régénérations restent inféodées aux pâturages des zones humides comme Mitragyna inermis, Machaerium lunatum et Antidesma venosum avec en moyenne 6 rejet pour $9 \mathrm{~m}^{2}$.

Dans l'ensemble, le taux de régénération des pâturages investigués lors des relevés de végétation n'est pas significatifs entre les pâturages à l'échelle de la zone d'étude $(\operatorname{Pr}=0,12)$ (Figure 10). Mais néanmoins les G3, G4, G5 semblent plus proches, les uns des autres par rapport au nombre de rejet. 


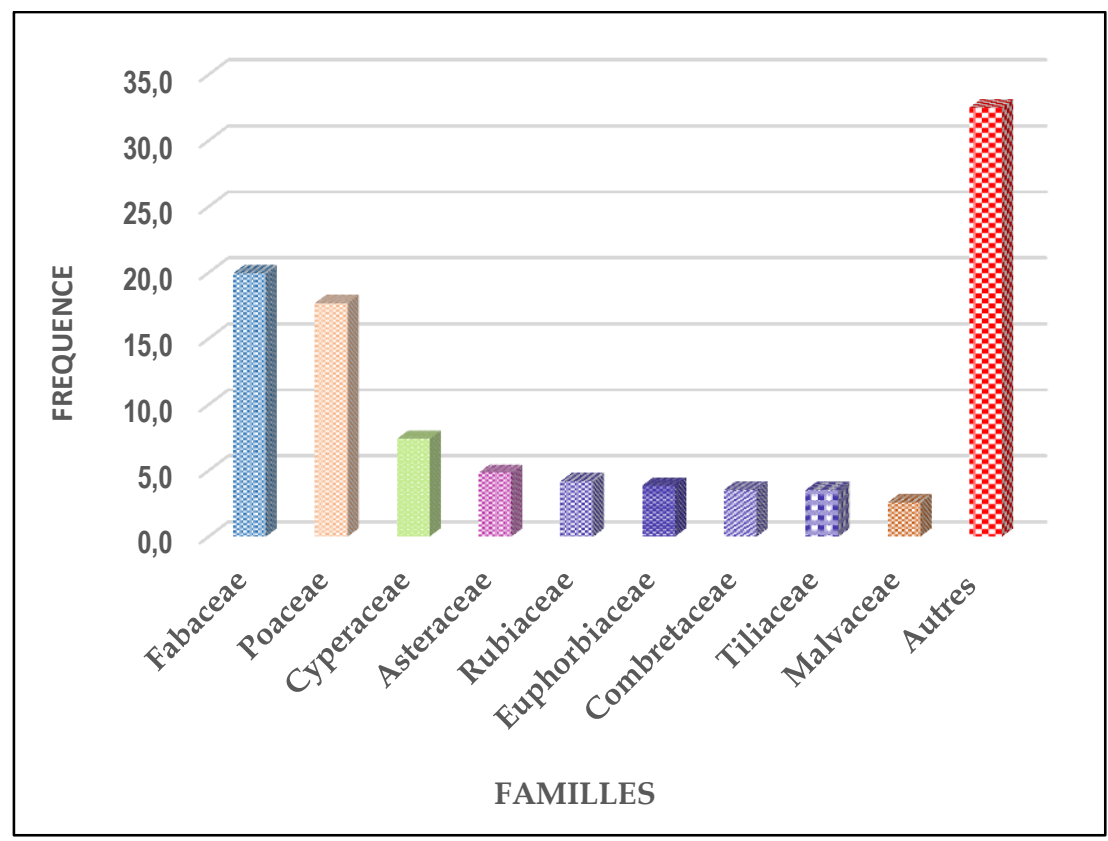

Figure 2 : Spectre des familles des espèces recensées.

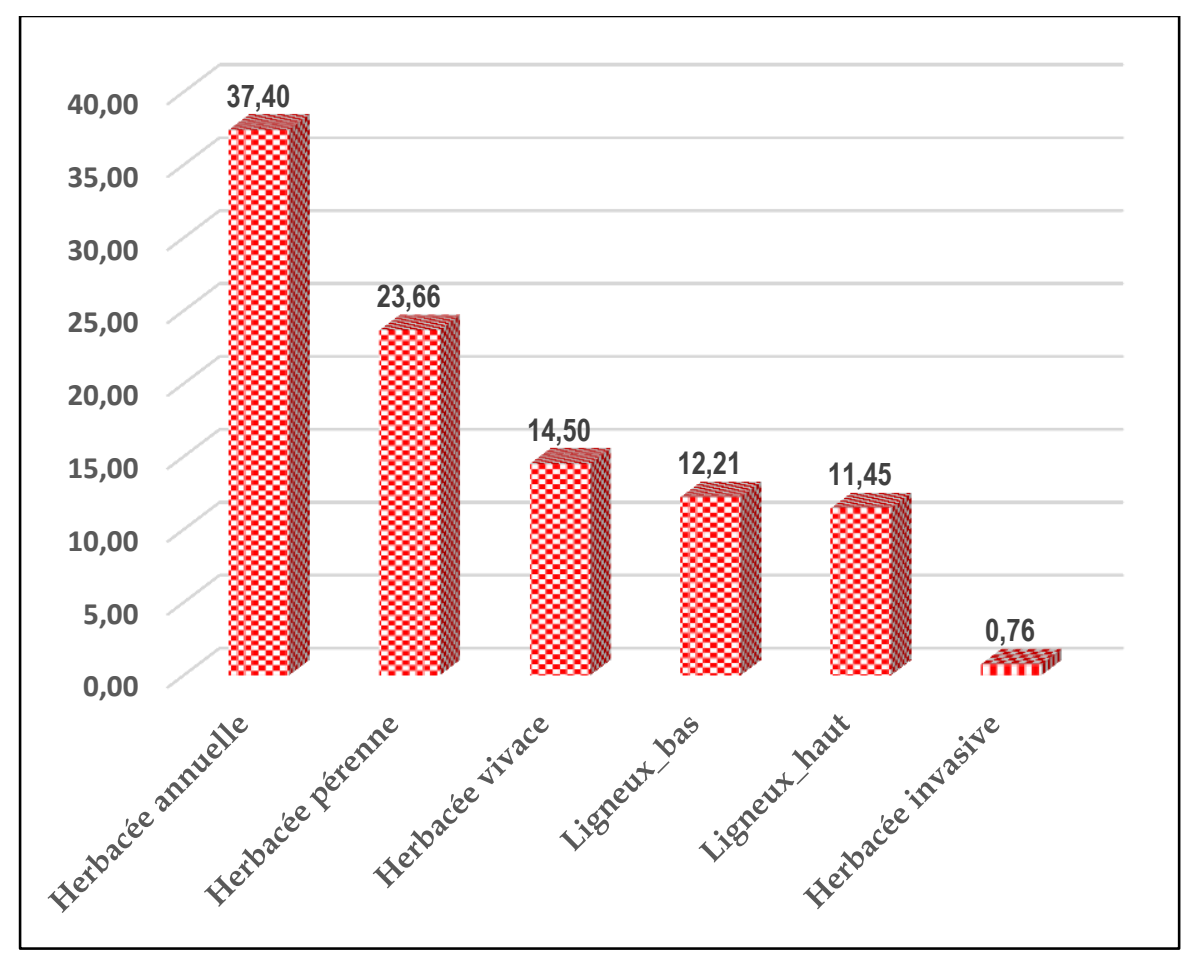

Figure 3 : Spectre des types floristiques des formations végétales pâturées (FVP). 


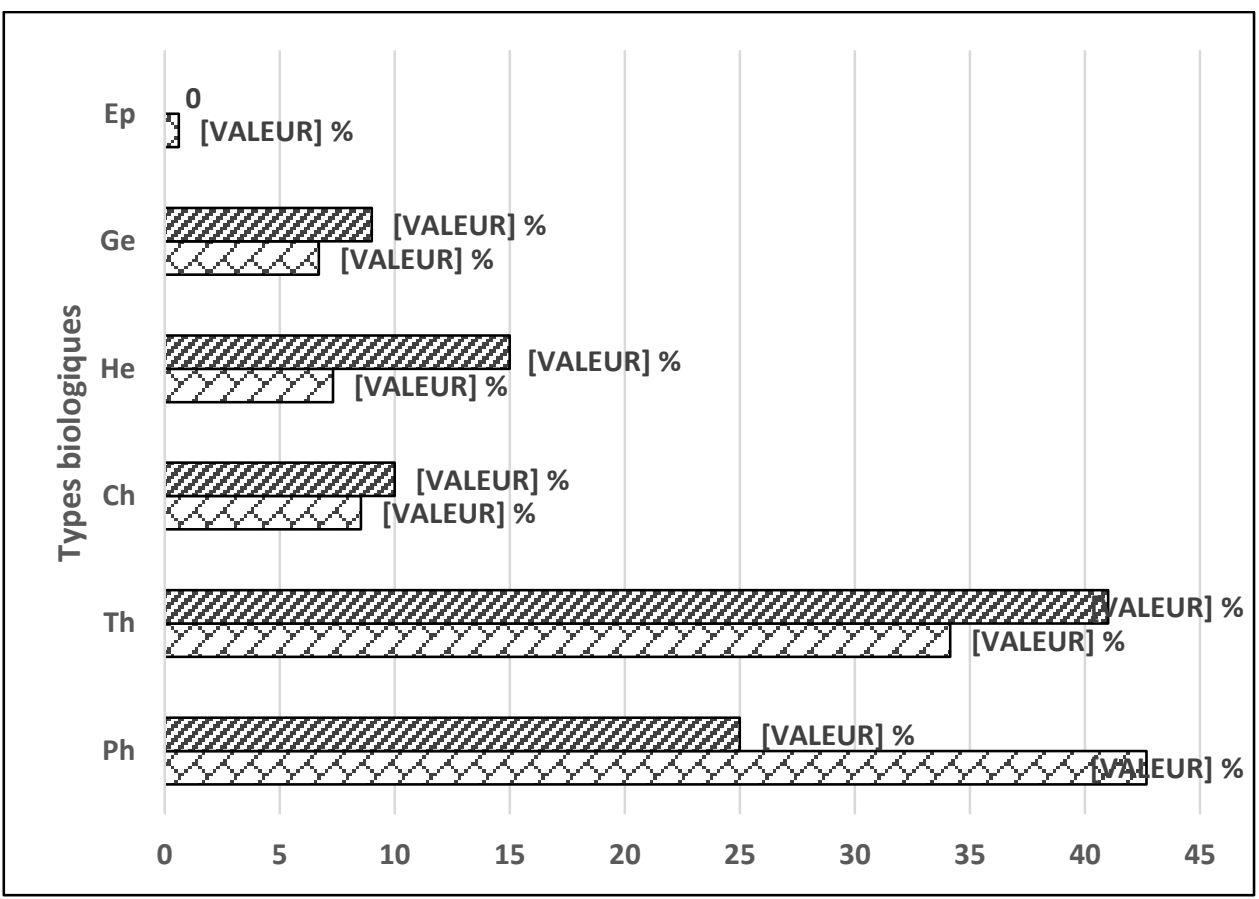

Figure 4 : Spectre des types biologiques des espèces fourragères et recensées ; $\mathrm{Ph}$ : phanérophytes ; Th : thérophytes ; Ch : chaméophytes ; Ge: géophytes; He: hémicriptophytes.

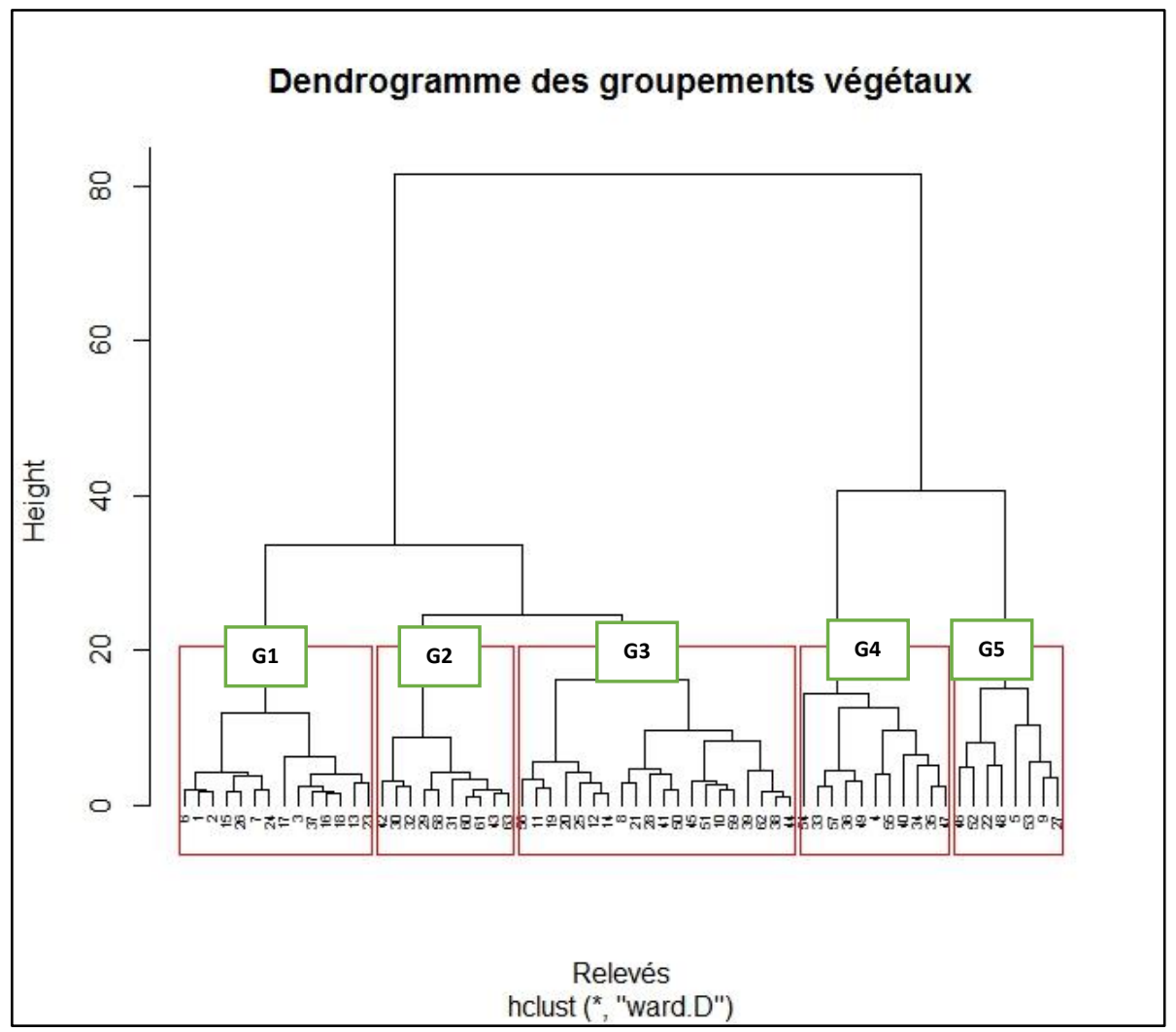

Figure 5: Dendrogramme des 63 relevés issus de la matrice phytosociologique. 


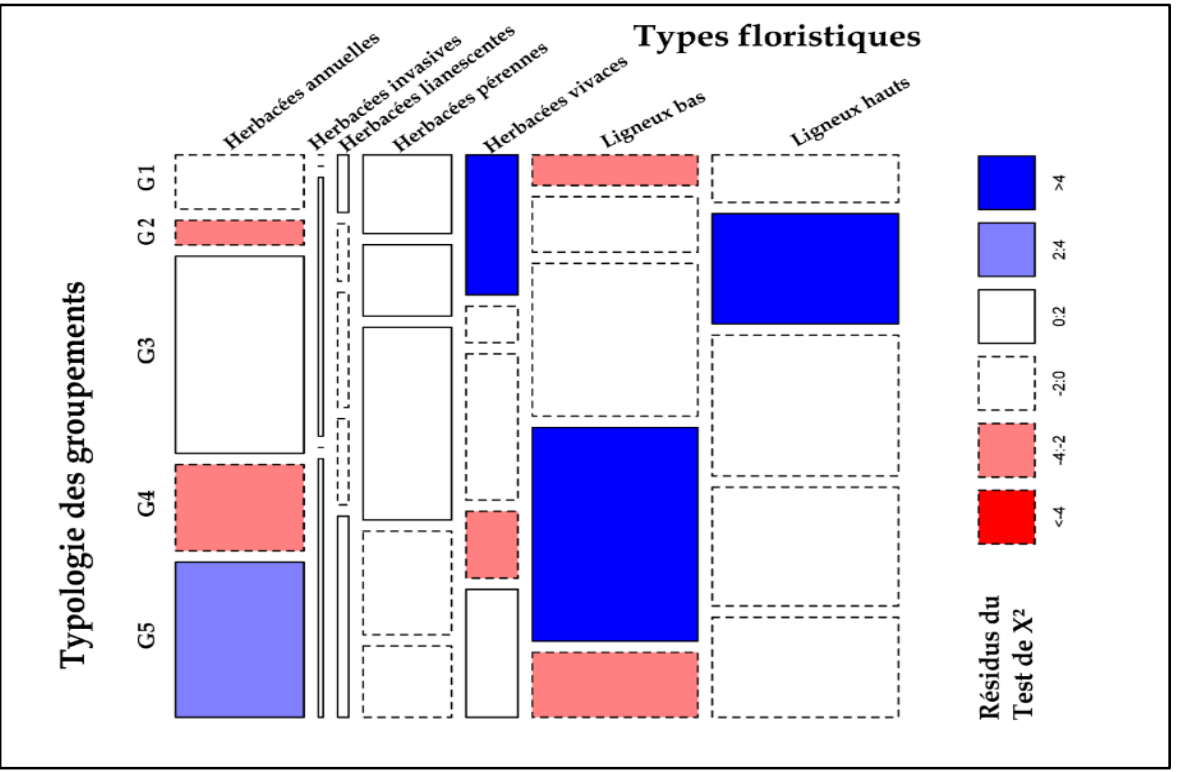

Figure 6: Spectre des types floristiques des groupements végétaux discriminés.

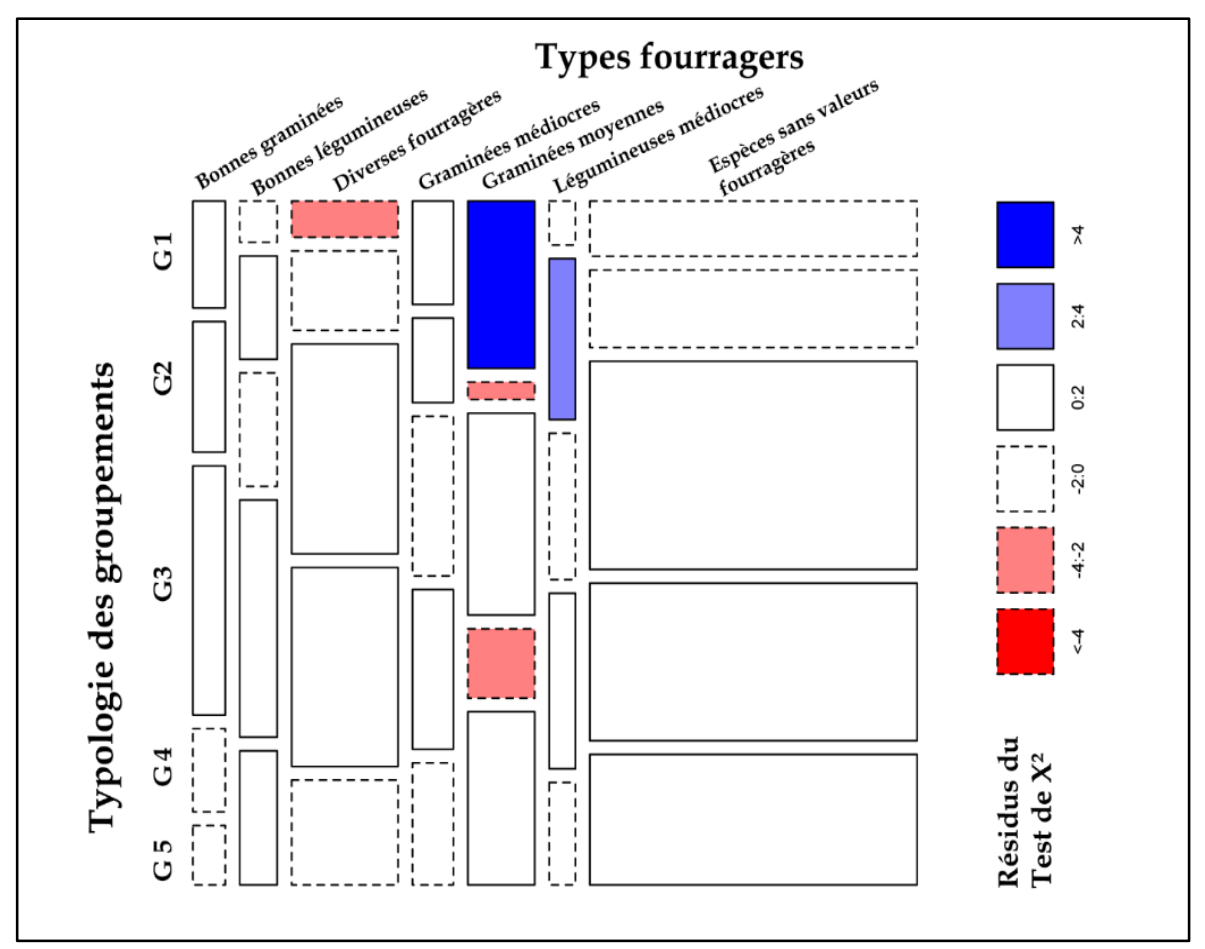

Figure 7: Spectre des types fourragers des groupements végétaux discriminés. 


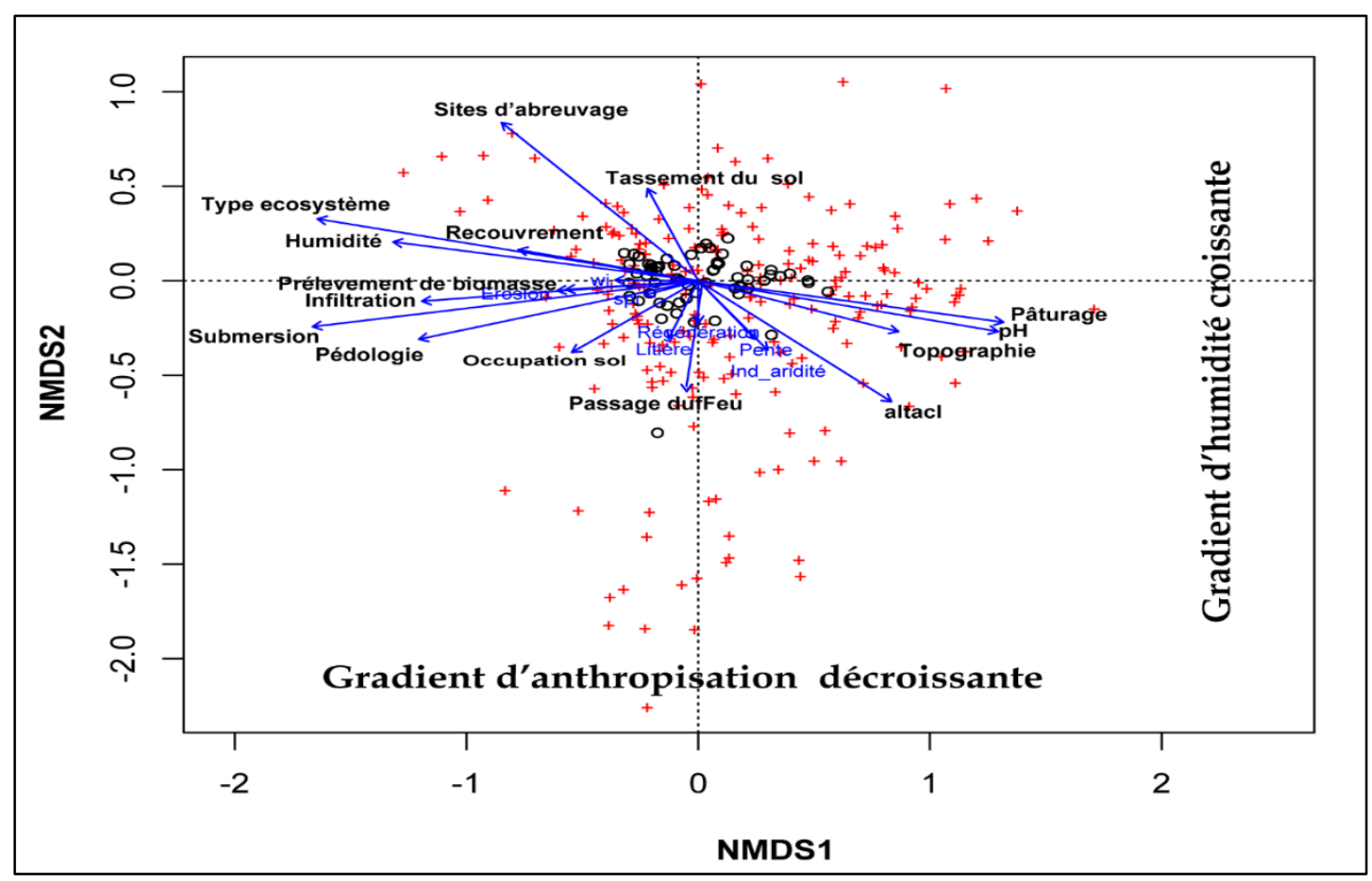

Figure 8. Ordination des déterminants influençant la distribution des espèces et relevés.

(Note : en noir les variables significatives, en texte bleu les variables non significatives; les cercles noirs et les croix rouges dénotent les relevés d'inventaires et les espèces recensées, respectivement).

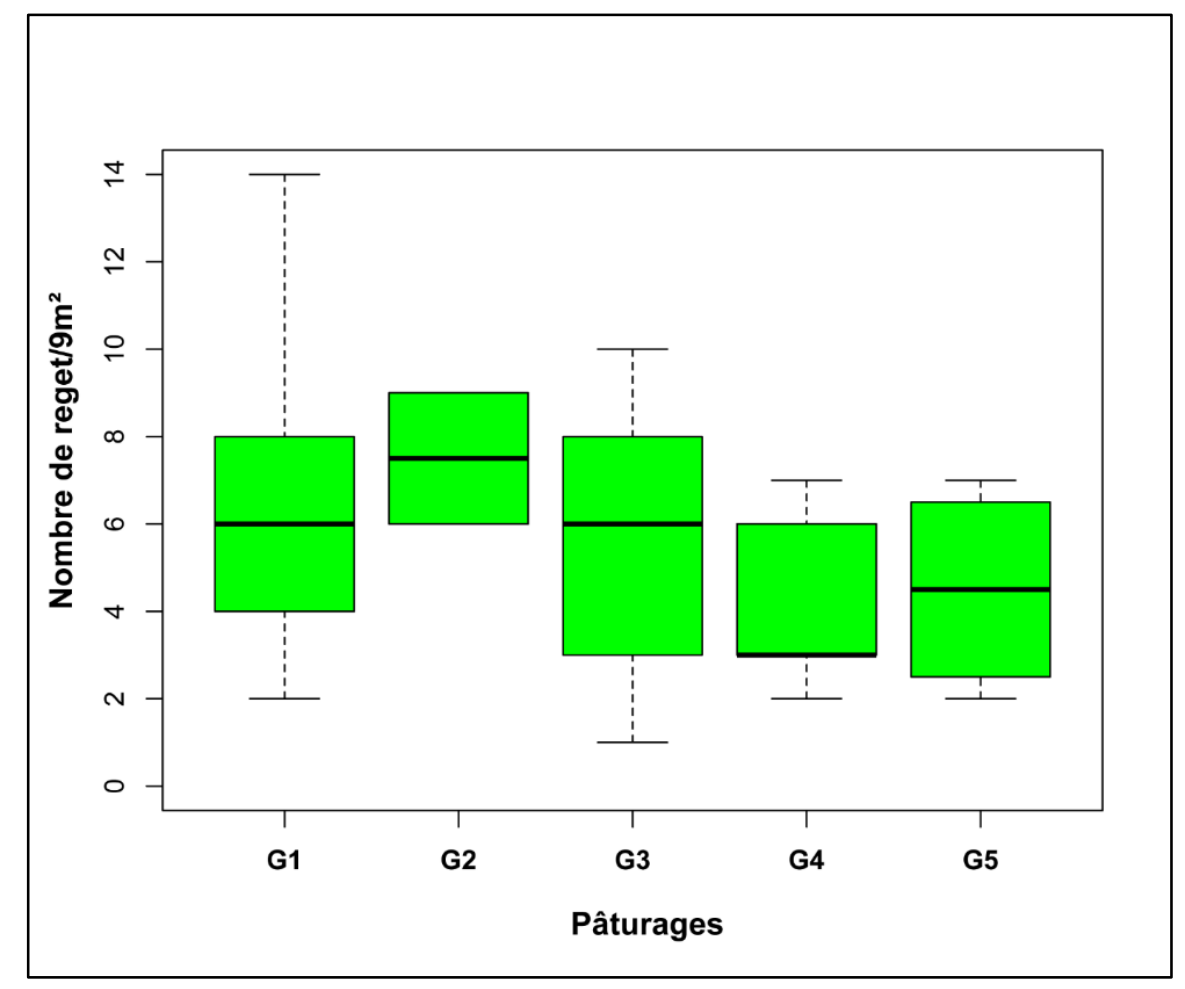

Figure 9: Variation du taux de régénération des pâturages discriminés. 


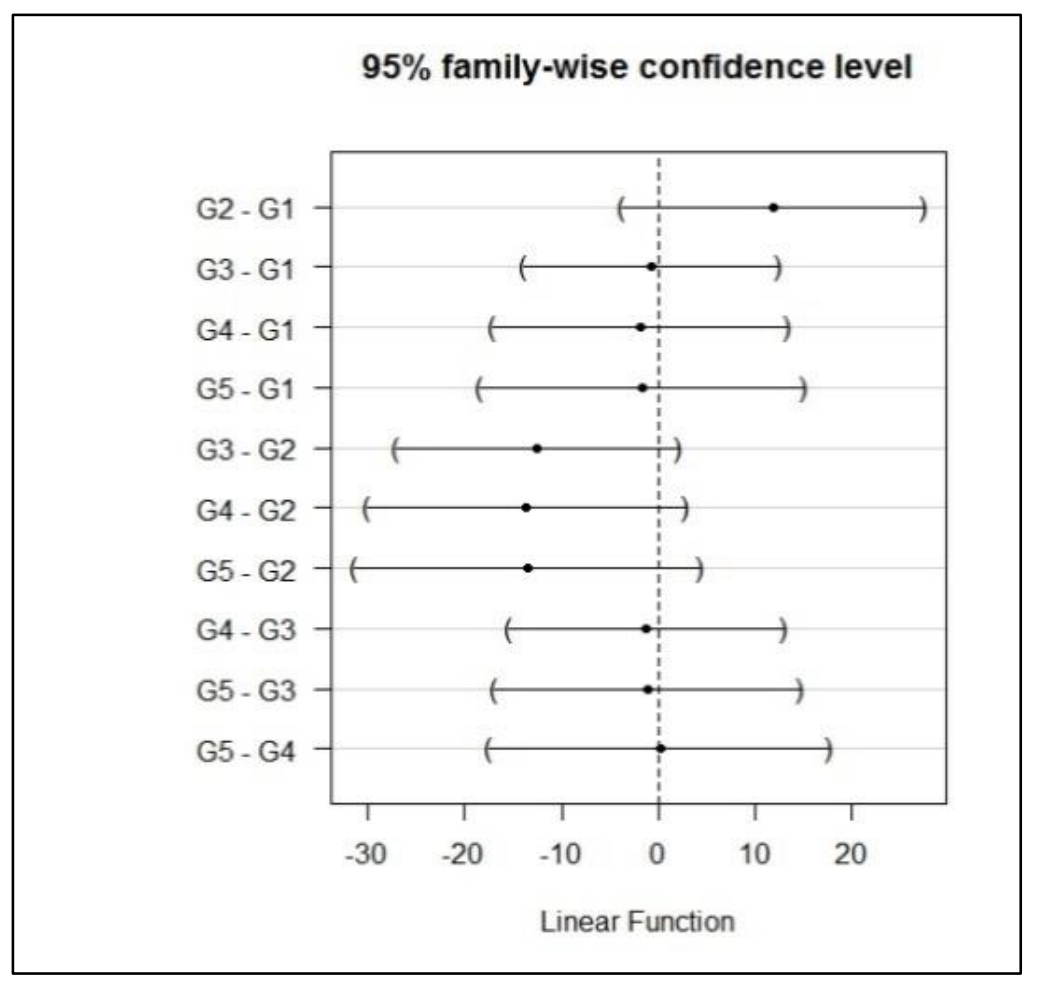

Figure 10 : corrélation entre les pâturages suivant leur taux de régénération.

\section{DISCUSSION}

\section{Analyse floristique et des variables écologiques}

Les formations végétales constituent la principale source d'alimentation du cheptel ruminant à travers la zone. La diversité floristique de ces formations reste le principal facteur directeur des mouvements pastoraux. En effet, à l'échelle de la zone, 4 principales familles (Fabaceae; Poaceae; Cyperaceae ; Asteraceae) sont les plus représentées. Cette cohorte de familles est constituée pour la plupart d'espèces fourragères dans une grande proportion. Cette richesse fourragère de ces familles constatées, montre que ces dernières constitueraient à l'échelle de la zone la principale source d'approvisionnement en fourrage pour le cheptel local et transhumant. Cette composition floristique des types végétaux serait un principal facteur directeur des éleveurs dans la fréquentation des formations végétales d'importance pastorales. La forte représentativité en fourrage de ces familles serait liée aux caractéristiques nutritives et à la disponibilité spatio temporelle des espèces qu'elles regroupent en leur sein. Cette richesse fourragère avait aussi été révélée par Lesse et al. (2016) dans les pâturages parcours pastoraux du centre du Bénin. Cette richesse floristique est supérieure aux investigations de Folega et al (2018) entreprises dans les aires de pâture abritant les inselbergs de la zone et à celle et à celle de Omourou et Lejoly (2003). L'indice de shannon (H') de la pâture à Mitragyna inermis et Cynodon dactylon est faible $(1,51)$ avec $\mathrm{E}=$ 0,37 par rapport aux indices des autres pâturages (Tableau 2). Ce faible indice de Pielou révèle une faible diversité floristique au sein dudit pâturage et serait expliqué par les perturbations anthropiques comme une pression du pâturage; l'occurrence des feux de végétation et justifierait le faible taux de régénération naturelle. D'autres facteurs écologiques liés à l'hydromorphie du sol et le degré de submersion très prolongé auraient 
des impacts négatifs sur la diversité floristique du pâturage et par conséquent serait un facteur limitant de cette faible diversité. Par contre les pâturages à Lonchocarpus sericeus Diheteropogon amplectens et Machaerium lunatum et Leersia hexandra ont respectivement 5,99 et 4,6 comme indice de shannon. Cette hausse de l'indice de ces pâturages traduit une forte richesse floristique de ces aires de pâture en herbacée pérenne, signe que les facteurs anthropiques et topoédaphiques n'ont pas fortement dégradés ces pâturages (Tableau 1).

\section{Analyse des types floristiques}

Les types de FVP discriminés sont constamment soumis aux perturbations anthropiques liées aux feux de végétation. $\mathrm{La}$ strate herbacée des pâturages de la zone est caractérisée par une flore riche en herbacées pérennes comme le genre Brachiaria; Panicum, Diheteropogon. Ces espèces très abondantes constituent le fourrage mais peuvent aussi être de potentielle sources de litière vecteur de la propagation de feu de brousse. Cette occurrence de feux de végétation dans les aires de pâture expliquerait le faible taux de régénération naturelle observable à l'échelle de la zone et aurait un effet néfaste sur la diversité floristique dans les FVP. La prépondérance du feu sur les pâturages traduit aussi une dégradation des types de végétation.

La biomasse des FVP est composée de $76 \%$ d'herbacée et 24 pour les ligneux. Cela suppose que les herbacées quelle que soit leur nature, restent la biomasse la plus sollicitée par le cheptel ruminant dans la zone. Les herbacées annuelles sont les plus représentées suivies des herbacées pérenne et vivace. Cela serait dû à leur valeur nutritive et à leur productivité sur les aires de pâture. La faible proportion des ligneux serait liée aux facteurs anthropiques (feu de végétation associés à la pression du pâturage aux activités anthropiques) sur la régénération naturelle de ces aires de pâture. Alkemade et al. (2013) ont relevé ces mêmes impacts de la pression pastorale sur la diversité végétale dans d'autres écosystèmes pâturés. Ces derniers sont aussi utilisés par les cheptels durant les périodes de soudures. Des auteurs comme Sèwadé et al. (2016) ont révélé le potentiel nutritionnel de certains ligneux bas (Flueggya virosa) recensés dans le cadre de cette étude

Les formes biologiques des pâturages montrent une dominance de phanérophytes (23\%) suivies des thérophytes (18\%). Ce recouvrement des phanérophytes et des thérophytes traduit une dégradation des FVP investiguées. Ceci serait lié à la pression pastorale, aux feux de végétation, aux autres formes d'activités anthropiques et aux conditions climatiques. Des résultats similaires ont été obtenus par Daget et Poisonnet (2010) qui incriminent cette prolifération de thérophytes aux conditions biotiques. Cette variabilité floristique expliquerait les mouvements migratoires des éleveurs et des conflits entre les éleveurs et les populations locales dans la zone.

\section{Analyse des déterminants de la diversité des FVP}

Sur la figure 8 , on observe que le pâturage et les activités anthropiques sont localisés dans deux plans factoriels différents. Ceci traduit une corrélation entre les deux facteurs et montre que, outre le pâturage, les autres formes d'anthropisation (coupes de bois, installation des foyers de carbonisation, etc.) contribuent à la dégradation des pâturages de la zone. Ce qui supposerait que les activités anthropiques sont des paramètres vecteurs de la perte de la diversité floristique et de la dégradation des pâturages. Les mêmes constats ont été observés entre la régénération et le degré de submersion du pâturage. Une forte régénération serait un signe de dégradation des pâturages de même qu'une submersion prolongée (Tableau 2).

A l'échelle de la zone d'étude, la régénération naturelle dans les différents types de pâturages discriminés reste faible. Le rejet de souche naturelle demeure l'unique mode de régénération rencontrée dans ces formations végétales. Les principaux rejets sont observés chez les espèces inféodées aux zones très humides comme Mitragyna inermis; Machaerium lunatum qui sont plus résistantes aux feux de végétation et très peux sollicitées 
comme fourrage mais utilisées comme bois énergie par les populations locales. Les espèces à très faibles rejet sont Lonchocarpus sericeus et Daniellia oliveri dont les feuilles sont utilisées comme fourrage par le cheptel. Ce faible taux de régénération observé dans les aires de pâture traduit une dégradation des pâturages due aux pressions du pâturage et des autres formes de pressions anthropiques (prélèvement $\mathrm{du}$ bois énergie, foyer de fabrication de charbon de bois), l'impact des feux de végétation. Wala et al. (2012) ont relevé les mêmes formes de pressions sur la diversité végétale dans les formations végétales similaires de la région centrale au Togo.

Tableaux 1 : Récapitulatif des indices de diversité des pâturages discriminés dans la zone.

\begin{tabular}{lccc}
\hline Pâturages & S & H' $^{\prime}$ & E \\
\hline G1 & 61 & 1,51 & 0,37 \\
G2 & 69 & 2,83 & 0,36 \\
G3 & 169 & 2,97 & 0,29 \\
G4 & 128 & 5,99 & 0,31 \\
G5 & 143 & 4,6 & 0,30 \\
\hline
\end{tabular}

Tableaux 2 : Récapitulatif des variables écologiques directrices de la dégradation des FVP.

\begin{tabular}{|c|c|c|c|c|c|c|}
\hline Facteurs directeurs & Déterminants & NMDS1 & NMDS2 & $\mathbf{r} 2$ & $\operatorname{Pr}(>\mathbf{r})$ & $\begin{array}{l}\text { Seuil de } \\
\text { significativité }\end{array}$ \\
\hline & Topographie & 0,874 & $-0,485$ & 0,139 & 0,008 & $* *$ \\
\hline & $\begin{array}{l}\text { Tassement du } \\
\text { sol }\end{array}$ & $-0,437$ & 0,899 & 0,057 & 0,162 & ns \\
\hline & Infiltration & $-0,982$ & $-0,189$ & 0,274 & 0,001 & $* * *$ \\
\hline & Pédologie & $-0,949$ & $-0,314$ & 0,273 & 0,002 & $* *$ \\
\hline & Erosion & $-0,958$ & $-0,286$ & 0,071 & 0,127 & ns \\
\hline \multirow[t]{4}{*}{ Topo-édaphiques } & Submersion & $-0,997$ & $-0,074$ & 0,500 & 0,001 & $* * *$ \\
\hline & pH & 0,939 & $-0,344$ & 0,336 & 0,001 & $* * *$ \\
\hline & Humidité & $-0,956$ & 0,292 & 0,337 & 0,001 & $* * *$ \\
\hline & Indice d'aridité & 0,772 & $-0,636$ & 0,030 & 0,406 & $\mathrm{~ns}$ \\
\hline
\end{tabular}




\begin{tabular}{|c|c|c|c|c|c|c|}
\hline & Altacl & 0,826 & $-0,563$ & 0,189 & 0,003 & $* *$ \\
\hline & Pente & 0,653 & $-0,757$ & 0,024 & 0,489 & $\mathrm{~ns}$ \\
\hline & spi & $-0,960$ & $-0,281$ & 0,011 & 0,744 & ns \\
\hline & WI & $-0,991$ & 0,133 & 0,023 & 0,527 & ns \\
\hline \multirow{4}{*}{ Anthropiques } & Feu & 0,061 & $-0,998$ & 0,058 & 0,159 & $* *$ \\
\hline & $\begin{array}{l}\text { Prélèvement de } \\
\text { biomasse }\end{array}$ & $-0,994$ & 0,111 & 0,064 & 0,153 & ns \\
\hline & Pâturage & 0,962 & $-0,273$ & 0,347 & 0,001 & $* * *$ \\
\hline & Occupation sol & $-0,770$ & $-0,638$ & 0,079 & 0,090 & $* *$ \\
\hline \multirow{5}{*}{ Environnementaux } & Recouvrement & $-0,965$ & 0,262 & 0,123 & 0,015 & $*$ \\
\hline & $\begin{array}{l}\text { Type } \\
\text { écosystème }\end{array}$ & $-0,991$ & 0,135 & 0,519 & 0,001 & $* * *$ \\
\hline & $\begin{array}{l}\text { Présence de } \\
\text { litière }\end{array}$ & $-0,344$ & $-0,939$ & 0,013 & 0,685 & ns \\
\hline & $\begin{array}{l}\text { Site } \\
\text { d'abreuvage }\end{array}$ & $-0,725$ & 0,689 & 0,236 & 0,001 & $* * *$ \\
\hline & Régénération & 0,341 & $-0,940$ & 0,005 & 0,842 & ns \\
\hline
\end{tabular}

Note : ns non significatif aux taux utilisés ; Code de significativité : $\quad 0^{‘ * * * ’} 0,001$ ‘**’ 0,01 ‘*’ 0,05 ‘’ 0,1 ‘’ 1

\section{Conclusion}

La présente étude menée dans le bassin du Mono a permis de montrer que l'élevage dans la zone reste tributaire de la composition floristique des formations végétales pâturées, des variables topo-édaphique et écologiques sous leurs diverses formes. L'analyse de variance a révélé que les activités anthropiques, la pression du pâturage, l'impact du feu, le degré de submersion, la topographie et la couverture du sol sont les facteurs environnementaux qui orientent la diversité floristique et la distribution des espèces dans les formations végétales pâturées. Les investigations phytosociologiques réalisées ont permis de discriminer cinq types de pâturage présentant différents indices de diversité et un taux de régénération relativement faible : le pâturage à Mitragyna inermis et Cynodon dactylon, le pâturage à Anogeissus leiocarpa et à Panicum maximum, le pâturage à Mitragyna inermis et
Paspalum orbiculare, le pâturage à Lonchocarpus sericeus et Diheteropogon amplectens et le pâturage à Machaerium lunatum et Leersia hexandra. Les Fabaceae, Poaceae, Combretaceae et Cyperaceae sont les familles les plus rencontrés dans ces pâturages. Les herbacées annuelles, pérennes et vivaces constituent l'essentiel de la biomasse et sources de fourrages pour le cheptel local et transhumant. La dominance des phanérophytes et des thérophytes dans le recouvrement des aires de pâture traduit une dégradation des pâturages, conséquence liée à la pression du pâturage et des feux de végétation. La régénération naturelle des ligneux à l'échelle reste faible. Seules les espèces ligneuses inféodées aux pâturages des humides comme Mitragyna inermis; Machaerium lunatum présentent 6 à 8 espèces pour $9 \mathrm{~m}^{2}$ à cause de l'occurrence des feux de végétation et la pression du pâturage. Cette situation montre les difficultés auxquelles font 
face les éleveurs dans leur recherche de fourrage. Cette pression diversiforme exercée par coexistence de différents facteurs continue de porter à faux la biodiversité fourragère et l'évaluation de la biomasse à l'échelle de la zone.

\section{CONFLIT D'INTERETS}

Les auteurs à l'unanimité déclarent qu'ils n'ont aucun conflit d'intérêt pour la publication de ce manuscrit.

\section{CONTRIBUTIONS DES AUTEURS}

$\mathrm{MD}, \mathrm{SA}, \mathrm{KW}$ ont apporté leur aide pour le plan d'élaboration et la lecture de ce manuscrit. BD, WA, KMLA ont participé à la collecte, l'analyse et le traitement des données de terrain tandis que $\mathrm{KB}$ et $\mathrm{KA}$ ont contribué aux orientations scientifiques de ce manuscrit.

\section{REMERCIEMENTS}

La présente étude est une partie d'un mémoire de thèse de doctorat développée à Université de Lomé (Togo). Les auteurs remercient le Laboratoire de Botanique et Ecologie Végétale (Université de Lomé, Togo) pour avoir accepté héberger cette thèse et pour le support lors de la collecte des données sur le terrain. Ils témoignent aux sieurs Professeur Djaneye-Boundjou Gbandi, Laboratoire la chimie des Eaux et Aklikokou Kodjo (Laboratoire de Physiologie Animale) pour leur soutien moral et technique durant la collecte et le traitement de données. Toute notre profonde gratitude aux reviewers inconnus pour leurs sacrifices et leur importante contribution à ce document. Ils remercient vivement aussi les auteurs anonymes et les initiateurs du projet $\mathrm{R}$ dont les scripts ont servi aux traitements des données.

\section{REFERENCES}

Adjonou K, Djiwa O, Kombaté Y, Kokutse AD, Kokou K. 2010. Etude de la dynamique spatiale et structure des forêts denses sèches reliques du Togo: implications pour une gestion durable des aires protégées. Int. J. Biol. Chem. Sci., 4: 168-183. DOI: doi.org/10.4314/ijbcs.v4i1.54242.
Afidégnon D. 1999. Les mangroves et les formations associées du Sud-Est du Togo : Analyse éco-floristique et cartographie par télédétection spatiale. Département de Botanique et Ecologie Végétale. Th. Doct., Univ. Bénin, Togo 237 p.

Agonyissa D, Sinsin B. 1998. Productivité et capacité de charge des pâturages naturels du Bénin Revue Elev.Méd.vét.Pays Trop., 51: 239-246.

Alkemade RSR, Reid M, Van den Berg J, De Leeuw JM. 2013. Assessing the impacts of livestock production on biodiversity in rangeland ecosystems. Rangeland Ecology \& Management, 110: 2090020905. Doi: $10.1073 /$ pnas.1011013108.

Aubreville A. 1957. Accord à yangambi sur la nomenclature des types africains de vegetation. Bois et Forets des Tropiques, 51: 22-27.

Bechir AB, Mopate LY, Kabore-Zoungrana CY. 2009. Evaluation de la disponibilité saisonnière du fourrage ligneux en zone soudanienne. Int. J. Biol. Chem. Sci., 3(1): 135-146.

Braun-Blanquet J. 1932. Plant Sociology. Edn Mc Gray Hill: New York, London.

CEDEAO CO. 2008. Elevage et marché régional au Sahel et en Afrique de l'Ouest: potentialités et défis. In: Afrique de l'Ouest/OCDE. CEDEAO CO.

Correra A. 2006. Dynamique de l'utilisation des ressources fourragères par les dromadaires des pasteurs nomades $d u$ parc national $d u$ banc d'arguin (Mauritanie). Département, Ecologie et gestion de la biodiversité, UMS 2699: Inventaire et suivi de la biodiversité , $362 \mathrm{p}$.

Daget P, Possonet J. 2010. Prairies et Pâturages: Méthodes d'Etude de Terrain et Interprétations. CIRAD; $986 \mathrm{p}$.

Demakou Y, Dourma M, Akpavi S, Atato A, Tchamie TTK. 2012 Typologie des principaux groupements végétaux des savanes soudaniennes semi-arides au nord Togo. Rev. Sc. Env. Univ., Lomé (Togo) $9 \mathrm{p}$.

Demakou Y, Dourma M, Woegan AY, Akpavi S, Atato A, Wala K, Batawila K, Tchamie TTK, Akpagana K. 2011. Contribution a la connaissance de 
quelques paysages vegetaux des savanes soudaniennes semi arides au Togo. J. Rech. Sci. Univ. Lomé (Togo), 13: 85-93. Dimobe K, Wala K, Dourma M, Kiki M, Woegan YA, Folega F, Batawila K, Akpagana K. 2014. Disturbance and population structure of plant communities in the wildlife reserve of Oti-Mandouri in Togo (West Africa). Annu. Res. Rev. Biol., 4: 2501-2516.

Direction des Statistiques Animales du Burkina Faso. 2008. Les Statistiques du Secteur de l'Elevage au Burkina Faso. Direction des Statistiques Animales du Burkina Faso; 124.

Diwediga B, Wala K, Folega F, Dourma M, Woegan AY, Akpagana K, Le BQ. 2015. Biophysical and anthropogenous determinants of landscape patterns and degradation of plant communities in Mo hilly basin (Togo). Ecological Engineering, 85: 132-143. Doi: 10.1016/j.ecoleng.2015.09.059.

Djenontin J. 2010. Dynamique des Stratégies et des Pratiques d'Utilisation des Parcours Naturels pour l'Alimentation des Troupeaux Bovins au Nord-Est du Bénin. Earth Sciences. Université de Abomey-Calavi; 275 p.

Dourma M. 2008 Les forets claires a Isoberlinia sp. dans la zone soudanienne du Togo: Ecology, Régénération naturelle et impacts humains. Département of Botanique. Université of Lomé 185 p.

Ern H. 1979. Die vegetation Togo. Gliederrung, Gefährdung, Erhaltung. Willdenowia, 9: 295-312.

FAO. 2011. Trois populations humaine, trois situations de sécurité alimentaire. L'élevage dans le monde en 2011 • Contribution de l'élevage à la sécurité alimentaire. FAO; 40 p.

Fousseni F, Kperkouma W, Agbelessessi YW, Madjouma K, Marra D, Komlan B et Koffi A. 2018. Flore et communautés végétales des inselbergs du Sud-Est du Togo. Physio-Géo, 12: 1-21.

Folega F, Zhan CY, Zhao XH, Wala K, Akpagana K. 2010a. Floristic diversity in most dry and environmentally disturbed areas of Northern Togo. International
Conference on Biology Environment and Chemistry, 241-243 p.

Gaoue OG, Sinsin B. 2003. Typologie et écologie des pâturages naturels de la zone cynégétique de la Pendjari (nordouest Bénin): éléments pour l'aménagement et la gestion rationnelle de l'habitat de la faune sauvage. Parakou, Bénin. 25-29 juin 2001. Faculté des Sciences Agronomiques, Université d'Abomey-Calavi, Bénin.

Gregor AC. 2001. The effects of Cattle Grazing on Vegetation Diversity and Structural Characteristics in the Semiarid Rangelands of North Queensland. Tropical Plant Sciences. James Cook University, School of Tropical Biology, James Cook University; 241 p.

Guelly KA, Kokou K, Afiademanyo K. 1997. Etude de quelques zones humides du Parc National de la Keran et du sud de la Réserve de Faune de Togodo. Rapport de fin de projet, Lomé, 81p.

Jürgens N. 1990. A life form concept including anatomical characters, adapted for the description succulents plants. Mitteilungen aus dem Institut für allgemeine Botanik Hamburg, 23: 321342.

Kiema A, Sawadogo I, Ouedraogo T, Nianogo AJ. 2012. Stratégies d'exploitation du fourrage par les éleveurs de la zone sahélienne du Burkina Faso. International Journal of Biological and Chemical Sciences, 6(4): 1492-1505. DOI: doi.org/10.4314/ijbcs.v6i4.8.

Kokou K, Caballé G. 2000. Les îlots forestiers de la plaine côtière togolaise. Bois et Forêts des Tropiques, 263(1): 39-51.

Krätli S, Hülsebusch C, Brooks S, Kaufmann B. 2013a. Pastoralism: a critical asset for food security under global climate change. Animal Frontiers, 2: 42-50.

Lejoly J, Sinsin B. 1991. Structure et valeur pastorale des pâturages soudaniens de bas-fonds dans le nord-Bénin. IVè Conférence Internationale des Terres de Parcours, Montpellier, France, 554-557 p.

Lesse P, Houinato M, Azihou F, Djenontin JBS. 2016. Typologie, productivité, capacité de charge et valeur pastorale des 
pâturages des parcours transhumants au Nord Est de la République du Bénin. International Journal of Innovation and Applied Studies, 14: 132-150.

MEA. 2005. Ecosystems and Human WellBeing: Synthesis. Island Press: W., DC, USA

Ministère de l'Agriculture et de l'Elevage, 2011. Document Stratégique de Réduction de la Pauvreté (DSRP). Ministère de l'Agriculture et de l'Elevage, $116 \mathrm{p}$.

Oumorou M, Lejoly J. 2003. Écologie, flore et végétation de l'inselberg Sobakperou (Nord-Bénin). Acta Botanica Gallica, 150(1): 65-84.

Peet RK, Wentwoth TR, White PS. 1998. A fexible, multipurpose method for recording vegetation composition and structure. Castanea, 63: 262-274.

Raunkiaer C. 1934. The Life Forms of Plants and Statistical Plant Geography. Oxford University Press: London; 632 p.

Sala OE, Oesterheld M, Leon RJC, Soriano A. 1986. Grazing effects upon plant community structure in subhumid grasslands of Argentina. Vegetation, 67: 27-32.

Sala OE, Paruelo JM. 1997. Ecosystem services in grasslands In Nature's Services: Societal Dependence on Natural Ecosystems, Daily GCE (Ed.). Island Press:Washington, DC.

Sala OE, Yahdjian L, Havstad K, Aguiar MR. 2017. Rangeland ecosystem services: nature's supply and humans' deman. In Rangeland Systems, Briske DD (Ed.). Springer; 467-489.

Shmida A. 1984. Whittaker's plant diversity sampling method. Israel Journal of Botany, 33: 41-46.

Sinsin B. 1993. Phytosociologie, écologie, valeur pastorale, productivité et capacité de charge des pâturages naturels du périmètre Nikki-Kalalé au Nord Bénin. Univ. Lib. de Bruxelles, Thèse de doctorat, Univ. Lib. de Bruxelles, Belgique.
Sinsin B. 1995. Les ressources fourragères du Togo et leur aménagement. TCP/TOG/4452, F. (Ed.), Série, Notes du Laboratoire d'Ecologie Appliquée.

Sokemawu K. 2008. Les systèmes pastoraux, dynamique des paysages et circuit de commercialisation du bétail dans la région des Savanes au Togo. Département de géographie. Université de Lomé, $342 \mathrm{p}$.

Stohlgren TJ. 2007. Measuring plant diversity. In Lessons from the Field. Press, O.U. : Oxford.

Sudhakar Reddy C, Hari Krishna PSLM, Ruchira B, Sharma KC. 2011. Composition of Life forms and Biological spectrum along climatic gradient in Rajastan, india. International Journal of Environmental Sciences, 1: 1632-1639.

Toutain B, Marty A, Bourgeot A, Ickowicz A, Lhoste P. 2012. Pastoralisme en zone sèche. Le cas de l'Afrique subsaharienne.

Union africaine. 2010. Cadre pour une Politique du Pastoralisme en Afrique: Sécuriser, Protéger et Améliorer les Vies, les Moyens d'Existance et les Droits des Communautés Pastorales. Département d'Economie Rurale et d'Agriculture: Addis-Abeba, Ethiopie.

Wala K, Woegan YA, Borozi W, Dourma M, Atato A, Batawila K, Akpagana K. 2012. Assessment of vegetation structure and human impacts in the protected area of Aledjo (Togo) Far. J. Ecol., 50: 355366.

Yameogo G, Kiema A, Yelemou B, Ouedraogo L. 2014. Caractéristiques des ressources fourragères herbacées des pâturages naturels du terroir de Vipalogo (Burkina Faso). International Journal of Biological and Chemical Sciences, 7(5): 2078-2091. Doi doi.org/10.4314/ijbcs.v7i5.25. 